\title{
Controle Químico DE Panicum repens E Paspalum repens ${ }^{1}$
}

\author{
Chemical Control of Panicum repens and Paspalum repens
}

CARBONARI, C.A. ${ }^{2}$, MARTINS, D. ${ }^{3}$, TERRA, M.A. ${ }^{4}$ e MARCHI, S.R. ${ }^{4}$

\begin{abstract}
RESUMO - Panicum repens e Paspalum repens são espécies infestantes de ambientes úmidos e alagados, freqüentes em margens de lagos, reservatórios, canais de irrigação e drenagem. O presente trabalho teve como objetivo avaliar a eficiência de diferentes herbicidas no controle dessas duas espécies. O experimento foi conduzido no Núcleo de Pesquisas Avançadas em Matologia, do Departamento de Produção Vegetal, pertencente à Faculdade de Ciências Agronômicas/UNESP, campus de Botucatu-SP. As plantas foram cultivadas em caixas d'água e as pulverizações foram realizadas utilizando-se um pulverizador costal pressurizado a $\mathrm{CO}_{2}$. Os tratamentos testados foram: glyphosate a 2.400, 3.360 e 4.320 g e.a. ha ${ }^{-1}+$ Aterbane $0,5 \% \mathrm{v} / \mathrm{v}$, glyphosate a $2.400,3.360$ e $4.320 \mathrm{~g} \mathrm{e.a.} \mathrm{ha}$. $^{-1}+$ Silwet $0.1 \% \mathrm{v} / \mathrm{v}$, imazapyr a 750 e $1.500 \mathrm{~g}$ e.a. ha-1 e diquat a 400 e 800 g i.a. ha h $^{-1}$ (em aplicações seqüenciais de 200+200 g i.a. ha-1 e 400+400 g i.a. ha ${ }^{-1}$ ), além de uma testemunha sem aplicação de herbicidas. O delineamento experimental utilizado foi inteiramente casualizado, com quatro repetições. Avaliações visuais de controle foram realizadas, sendo a massa seca das plantas determinada ao final do estudo. O herbicida glyphosate na dose de 4.320 g e.a. $\mathrm{ha}^{-1}$, independentemente do adjuvante utilizado, assim como na dose de 3.360 g e.a. ha-1 com Aterbane, proporcionou bom controle de Panicum repens e, em todas as doses e adjuvantes testados, promoveu controle excelente de Paspalum repens. O herbicida imazapyr, independentemente da dose testada, apresentou resultados insatisfatórios no controle das plantas de Panicum repens, porém promoveu um excelente controle de Paspalum repens; o herbicida diquat, apesar das duas aplicações e independentemente da dose utilizada, mostrou-se ineficiente para controle das duas espécies.
\end{abstract}

Palavras-chave: planta aquática, adjuvante, glyphosate, imazapyr, diquat.

\begin{abstract}
Two experiments were carried out to evaluate the susceptibility of Panicum repens and Paspalum repens Bergius to different herbicides applied in post-emergence. The plants were cultivated in reservoirs and the treatments tested were: glyphosate (Rodeo) at 2,400, 3,360 and 4,320 g a.e. $\mathrm{ha}^{-1}$ plus Aterbane 0.5\% v/v; glyphosate at 2,400, 3,360 and 4,320 g a.e. ha-1 plus Silwet 0,1\% v/v; imazapyr (Arsenal) at 750 and 1,500 g a.e. ha ${ }^{-1}$, diquat (Reward) at 400 and $800 \mathrm{~g}$ a.i. $\mathrm{ha}^{-1}$ (in sequential applications of $200+200 \mathrm{~g}$ a.i. $\mathrm{ha}^{-1}$ and $400+400 \mathrm{~g}$ a.i. $\mathrm{ha}^{-1}$, respectively) and an untreated control. A $\mathrm{CO}_{2}$ backpack sprayer with 2 bars and consumption of $200 \mathrm{~L} \mathrm{ha}^{-1}$ of spray solution was used.The experimental design was completely randomized with four repetitions. The control was evaluated visually and plant dry matter determined at the end of the study. The herbicide glyphosate at 4,320 g a.e. $\mathrm{ha}^{-1}$, regardless of the adjuvant used, and at 3,360 $\mathrm{g}$ a.e. $\mathrm{ha}^{-1}$ with Aterbane, provided a good control of Panicum repens, and an excellent control of Paspalum repens at all the doses tested; the herbicide imazapyr, regardless of the dose tested, presented unsatisfactory results in controlling Panicum repens plants, but provided an excellent control of Paspalum repens; the herbicide diquat was inefficient in controlling both species, despite the two applications and regardless of the dose applied.
\end{abstract}

Key words: aquatic plant, adjuvant, glyphosate, imazapyr, diquat.

Recebido para publicação em 9.2.2003 e na forma revisada em 10.9.2004.

Aluno de graduação, bolsista Fapesp, Departamento de Produção Vegetal da Faculdade de Ciências Agronômicas, Universidade Estadual Paulista - FCA-UNESP, Caixa postal 237, 18603-970 Botucatu-SP, <carbonari@ @ca.unesp.br>; ${ }^{3}$ Prof. Assistente de Doutor, Departamento de Produção Vegetal da FCA-UNESP; ${ }^{4}$ Eng.-Agr., Aluno de pós-graduação, Departamento de Produção Vegetal da FCA-UNESP.

Planta Daninha, Viçosa-MG, v. 22, n. 3, p. 453-460, 2004 


\section{INTRODUÇÃO}

Ambientes úmidos ou alagados, como várzeas ou margens de corpos hídricos, proporcionam condições ecológicas favoráveis à proliferação de diversas plantas daninhas, em razão do excesso de nutrientes causado pela eutrofização e sedimentação de partículas minerais e orgânicas, depositadas em função da erosão de áreas agrícolas mal manejadas e escoamentos de esgotos. Dentre as várias espécies de ocorrência nesse meio destacamse as gramíneas Panicum repens e Paspalum repens.

Panicum repens é uma espécie perene e Paspalum repens é anual, sendo ambas pertencentes à família Poaceae e de ampla importância e distribuição no Brasil (Kissmann, 1997; Lorenzi, 2000).

As duas espécies reproduzem-se por sementes e rizomas. Panicum repens produz grande quantidade de rizomas, que suportam longos períodos secos e adaptam-se facilmente à condição de alagamento, onde eles passam a flutuar (Moreira, 1977). Em razão de seu rápido desenvolvimento vegetativo, as plantas podem ocupar as margens de reservatórios rapidamente (Hoyer et al., 1996). Paspalum repens apresenta crescimento de seus ramos e rizomas a grandes distâncias para dentro d'água; eventualmente, eles se desprendem e passam a formar ilhas que continuam a crescer, chegando a atingir grandes extensões (Lorenzi, 2000).

As plantas daninhas de ambientes aquáticos são classificadas em diferentes grupos. As plantas marginais são espécies de terra seca, ou seja, ocorrem nas margens de canais, reservatórios e rios, aproveitando-se da umidade. Dessa forma, as sementes dessas espécies podem disseminar-se pelo curso d'água, atingindo grandes distâncias (Velini, 2000). Assim, apresentam-se como importantes infestantes de várzeas e canais de irrigação e de drenagem (Lorenzi, 2000). Também são encontradas em grande quantidade nas margens de lagos e reservatórios utilizados para múltiplos usos da água, como: geração de energia, recreação, pesca e navegação (Tanaka, 1998).

A ocorrência dessas plantas daninhas nos mais diversos ambientes aquáticos proporciona elevada perda d'água por transpiração, além de reduzir a velocidade da água nos canais, aumentando a sedimentação de partículas e encarecendo a manutenção destes (Oliveira Jr. \& Constantin, 2001). Pitelli (1998) afirma que essas espécies invadem represas rurais, facilitando o acúmulo de material erodido e reduzindo a qualidade e a capacidade de armazenamento de água, assim como a própria vida útil do reservatório.

Essas plantas marginais aumentam a incidência de insetos vetores de doenças, pois proporcionam um ambiente favorável à proliferação desses agentes. O controle dessas plantas daninhas pode fazer parte de uma estratégia de controle desses vetores, reduzindo o número de tratamentos utilizados no combate a esses insetos (Nir, 1988). Panicum repens é, ainda, um importante hospedeiro alternativo de patógenos, que são responsáveis por grandes danos à cultura do arroz (Kissmann, 1997).

No Brasil, apesar do imenso potencial hídrico e dos grandes danos causados por essas plantas, são escassos os trabalhos visando o seu controle. Segundo Martins (1998), entre os métodos de controle recomendados tem-se o controle químico. Atualmente os herbicidas mais utilizados no mundo são os seguintes: 2,4-D, glyphosate, diquat, endothal, compostos à base de cobre, fluridone e imazapyr.

Nir (1988), Shilling et al. (1990) e Reddy \& Singh (1992) testaram herbicidas no controle de Panicum repens e constataram grande potencial de controle do glyphosate e imazapyr. No entanto, no Brasil são escassos os trabalhos visando o controle de Panicum repens e Paspalum repens.

Esta pesquisa teve como objetivo avaliar a eficiência de alguns herbicidas aplicados em pós-emergência, isolados ou acrescidos de adjuvantes, no controle de Panicum repens e Paspalum repens.

\section{MATERIAL E MÉTODOS}

Os estudos foram instalados e conduzidos no Núcleo de Pesquisas Avançadas em Matologia (NUPAM), pertencente ao Departamento de Produção Vegetal da Faculdade de Ciências Agronômicas/UNESP, campus de Botucatu-SP. 
As plantas de Panicum repens e Paspalum repens foram cultivadas em caixas d'água de fibra de vidro com as dimensões de 60 x 60 x $45 \mathrm{~cm}$, contendo cerca de 40 litros de solo de várzea, o que proporcionou uma profundidade de aproximadamente $10 \mathrm{~cm}$. As características químicas do solo utilizado nos experimentos foram: $\mathrm{pH}\left(\mathrm{CaCl}_{2}\right)=4,7$; M.O. $\left(\mathrm{g} \mathrm{dm}^{-3}\right)=14 ; \mathrm{P}, \mathrm{B}$, $\mathrm{Cu}, \mathrm{Fe}, \mathrm{Mn}$ e $\mathrm{Zn}=41,0,21,8,5,338,34,6$ e $2,9 \mathrm{mg} \mathrm{dm}^{-3}$, respectivamente; e $\mathrm{H}+\mathrm{Al}, \mathrm{K}, \mathrm{Ca}$, $\mathrm{Mg}, \mathrm{SB}$ e CTC $=47,2,8,39,20,61$ e 108. As características físicas foram: $70 \%$ de areia, $23 \%$ de argila e $7 \%$ de silte. Foi realizada uma adubação de $250 \mathrm{~kg} \mathrm{ha}^{-1}$ da formulação comercial 04-14-08 antes do plantio e uma adubação nitrogenada de $60 \mathrm{~kg} \mathrm{ha}^{-1}$ com uréia 50 dias depois deste.

As plantas de Panicum repens foram coletadas no reservatório de Jaguaribe-SP, sendo colocadas três mudas em cada caixa d'água, após serem podadas cerca de $20 \mathrm{~cm}$ acima do colo. As plantas de Paspalum repens foram coletadas no reservatório de Barra Bonita-SP e reproduzidas através de estolões, sendo colocados sete por caixa d'água. Elas foram mantidas a pleno sol com uma lâmina d'água de 3 a $7 \mathrm{~cm}$ constante sobre o solo.

Os tratamentos utilizados nas duas espécies de plantas foram: glyphosate (Rodeo) a 2.400, 3.360 e 4.320 g e.a. ha ${ }^{-1}$ mais Aterbane a $0,5 \% \mathrm{v} / \mathrm{v}$; glyphosate a $2.400,3.360$ e 4.320 g e.a. ha ${ }^{-1}$; mais Silwet L77 0,1\% v/v; imazapyr (Arsenal) a 750 e 1.500 g e.a. ha ${ }^{-1}$ e diquat (Reward) a 400 e 800 g i.a. ha ${ }^{-1}$ (em aplicações seqüenciais de $200+200$ g i.a. ha ${ }^{-1}$ e 400+400 g i.a. ha ${ }^{-1}$, respectivamente). Manteve-se uma testemunha sem aplicação de herbicidas. O delineamento experimental utilizado foi o inteiramente casualizado, com quatro repetições.
Os herbicidas foram aplicados três meses após o plantio, quando as plantas de Panicum repens apresentavam uma altura entre 85 e $95 \mathrm{~cm}$ e as de Paspalum repens, entre 45 e $55 \mathrm{~cm}$. As aplicações seqüenciais dos tratamentos com o herbicida diquat foram realizadas aos seis dias após aplicação dos herbicidas, para Paspalum repens, e aos nove dias, para Panicum repens.

$\mathrm{Na}$ aplicação dos herbicidas foi usado um pulverizador costal, pressurizado a $\mathrm{CO}_{2}$ com pressão constante de 2 bar e equipado com um reservatório de 2 litros. O consumo de calda foi de $200 \mathrm{~L} \mathrm{ha}^{-1}$. A barra de aplicação foi equipada com dois bicos jato plano Teejet $110.02 \mathrm{XR}$, distanciados $50 \mathrm{~cm}$ entre si.

As condições climáticas no momento da aplicação dos herbicidas e da aplicação seqüencial dos tratamentos com o herbicida diquat estão apresentadas na Tabela 1 . As parcelas foram protegidas, com lonas plásticas, de possiveis rajadas de vento no momento da aplicação.

As avaliações de controle das plantas daninhas foram visuais, por meio de uma escala percentual de notas, em que 0 (zero) corresponde a nenhuma injúria demonstrada pela planta e 100 (cem) à morte das plantas, segundo a Sociedade Brasileira da Ciência das Plantas Daninhas (1995). Essas avaliações foram feitas aos $2,6,9,12,15,18,22,26,30$, 35,42, 49, 56 e 60 dias após aplicação dos herbicidas (DAA) para Panicum repens e aos 2 , $6,9,12,15,18,22,28,34$ e 38 DAA para Paspalum repens. Ao final das avaliações visuais foi avaliada a massa seca das plantas, cortando-se a parte aérea de todas elas e secando-as em estufa de circulação forçada de ar a $60^{\circ} \mathrm{C}$.

Tabela 1 - Condições climáticas no momento da aplicação dos herbicidas. Botucatu-SP, 2003

\begin{tabular}{|l|c|c|c|c|c|c|}
\hline \multirow{2}{*}{ Condição } & \multicolumn{2}{|c|}{ Aplicação dos herbicidas } & \multicolumn{4}{|c|}{ Reaplicação do diquat } \\
\cline { 3 - 7 } & & & Panicum repens & \multicolumn{3}{c|}{ Paspalum repens } \\
\cline { 2 - 7 } & Inicial & Final & Inicial & Final & Inicial & Final \\
\hline Horário da aplicação & $8 \mathrm{~h} 15$ & $9 \mathrm{~h} 25$ & $14 \mathrm{~h} 50$ & $14 \mathrm{~h} 50$ & $16 \mathrm{~h} 10$ & $16 \mathrm{~h} 20$ \\
\hline Umidade relativa do ar $(\%)$ & 56 & 54 & 48 & 48 & 54 & 55 \\
\hline Temperatura do ar $\left({ }^{\circ} \mathrm{C}\right)$ & 24,8 & 25,4 & 27,9 & 26,7 & 26,5 & 26,1 \\
\hline
\end{tabular}


Os parâmetros utilizados para o estabelecimento das notas foram: quantidade de plantas mortas, acúmulo de biomassa, inibição do crescimento, quantidade e uniformidade das injúrias e capacidade de rebrota das plantas.

Os resultados foram submetidos à análise de variância pelo teste $\mathrm{F}$, sendo as médias dos tratamentos comparadas pelo teste t a $5 \%$ de probabilidade para os resultados da avaliação visual e a $10 \%$ de probabilidade para aqueles da avaliação da massa seca.

\section{RESULTADOS E DISCUSSÃO}

Na Tabela 2 encontram-se os resultados das avaliações visuais de controle das plantas de Panicum repens, de 2 a 60 dias após a aplicação dos herbicidas.

Observa-se que no início das avaliações (2 DAA) as plantas submetidas à aplicação de glyphosate mostraram os sintomas mais severos dentre os herbicidas sistêmicos, apresentando um amarelecimento (clorose) nas folhas, que evoluiu durante o período experimental.

Ao final do estudo (60 DAA), os tratamentos na maior dose ( 4.320 g e.a. ha $\left.{ }^{-1}\right)$ com ambos os adjuvantes e na dose de 3.360 g e.a. ha ${ }^{-1}$ com Aterbane foram os que apresentaram o maior controle das plantas. Na maior dose de glyphosate, apesar de ao final do estudo não ter sido observada diferença significativa entre os surfatantes, o Silwet proporcionou controle mais eficiente durante grande período das avaliações.

Por sua vez, Shilling et al. (1990) testaram glyphosate com três surfatantes, em diferentes concentrações, no controle de Panicum repens e observaram diferenças tanto em função dos surfatantes como das doses. Esses resultados não corroboram com os obtidos, talvez pela dose inferior utilizada pelo pesquisador, bem como pelo estágio de crescimento das plantas no momento da aplicação, que foi bem juvenil. Reddy \& Singh (1992) também testaram os efeitos de adjuvantes organossiliconados, a fim de potencializar o controle de Panicum repens com glyphosate, e os compararam com outro surfatante nãoiônico, observando maior controle das plantas nos tratamentos com os organossiliconados, o que não foi aqui observado.

Para o herbicida imazapyr, leves sintomas foram observados em Panicum repens aos 2 DAA, porém apenas aos 12 DAA os sintomas evoluíram de clorose para necrose. No entanto, as duas doses (750 e 1.500 g e.a. ha-1) apresentaram controles insatisfatórios e semelhantes durante todo o período experimental.

Nir (1988), por sua vez, testou o herbicida imazapyr nas doses de 1.000 e 1.500 g e.a. ha ${ }^{-1}$ no controle de Panicum repens e em algumas outras plantas daninhas perenes de ocorrência em canais de drenagem, com resultados positivos. Esses resultados contraditórios podem estar relacionados à densidade de plantas e ao estádio de desenvolvimento destas, os quais não são citados nesse trabalho.

O herbicida diquat, aos 2 DAA, apresentou controle bastante expressivo, sendo este maior para a concentração de $400 \mathrm{~g}$ i.a. ha ${ }^{-1}$. Os sintomas aumentaram e evoluíram aos 6 DAA, quando as plantas apresentavam suas folhas bastante danificadas por necroses. Esse rápido desenvolvimento dos sintomas pode ser atribuído ao fato de o diquat ser um herbicida nãoseletivo e de contato, ou seja, que apresenta translocação bastante limitada na planta, pois interfere no processo de captação de luz solar e causa morte rápida dos tecidos (Rodrigues \& Almeida, 1998). Segundo Oliveira Jr. \& Constantin (2001), esse produto apresenta como característica uma rápida absorção seus sintomas são observados quase que de imediato, podendo ocorrer a morte das plantas em um a dois dias. A atividade e velocidade de ação deste herbicida são proporcionais à intensidade luminosa.

A partir dos 9 DAA, as plantas começaram a apresentar rebrotas, o que promoveu uma redução no controle. Aos 12 DAA, os sintomas passaram a evoluir novamente em função da aplicação seqüencial efetuada, tornando-se severos e semelhantes aos da primeira aplicação. A partir dos 15 DAA, novamente surgiram rebrotas, as quais progrediram até o final do estudo, comprometendo o controle. A partir dos 18 DAA, ambas as doses proporcionaram controle semelhante. 
Tabela 2 - Avaliações visuais de controle de plantas de Panicum repens em diferentes épocas após aplicação de herbicidas. Botucatu-SP, 2003

\begin{tabular}{|c|c|c|c|c|c|c|c|c|c|c|c|c|c|c|}
\hline \multirow{2}{*}{ Tratamento } & \multirow{2}{*}{\begin{tabular}{|c|} 
Dose \\
g i/e.a. ha ${ }^{-1}$
\end{tabular}} & \multicolumn{13}{|c|}{ Dias após a aplicação dos herbicidas (DAA) } \\
\hline & & 2 & 6 & $9^{3 /}$ & 12 & 15 & 18 & 22 & 28 & 35 & 42 & 49 & 56 & 60 \\
\hline 1. testemunha & - & $0,0 \mathrm{f}$ & $0,0 \mathrm{e}$ & $0,0 \mathrm{f}$ & $0,0 \mathrm{f}$ & $0,0 \mathrm{f}$ & $0,0 \mathrm{f}$ & $0,0 \mathrm{e}$ & $0,0 \mathrm{~d}$ & $0,0 \mathrm{e}$ & $0,0 \mathrm{e}$ & $0,0 \mathrm{f}$ & $0,0 \mathrm{e}$ & $0,0 \mathrm{f}$ \\
\hline 2. glyphosate $+\mathrm{At}^{1 /}$ & 2.400 & $15,3 \mathrm{dc}$ & $23,0 \mathrm{c}$ & $27,3 \mathrm{~d}$ & $40,7 \mathrm{~d}$ & $46,7 \mathrm{~d}$ & $48,7 \mathrm{~d}$ & $60,3 \mathrm{bc}$ & $72,7 \mathrm{~b}$ & $74,0 \mathrm{~b}$ & $77,0 \mathrm{~b}$ & $80,7 \mathrm{c}$ & $85,0 \mathrm{~b}$ & $85,3 \mathrm{c}$ \\
\hline 3. glyphosate + At & 3.360 & $18,0 \mathrm{~cd}$ & $23,0 \mathrm{c}$ & $29,8 \mathrm{~cd}$ & $42,8 \mathrm{~cd}$ & $48,3 \mathrm{~d}$ & $51,0 \mathrm{~d}$ & $58,8 \mathrm{bc}$ & $76,5 \mathrm{~b}$ & $80,8 \mathrm{~b}$ & $84,8 \mathrm{~b}$ & $87,8 \mathrm{ab}$ & $89,3 \mathrm{~b}$ & $90,3 \mathrm{ab}$ \\
\hline 4. glyphosate + At & 4.320 & $18,3 \mathrm{c}$ & $25,5 \mathrm{c}$ & $32,8 \mathrm{bcd}$ & $43,3 \mathrm{~cd}$ & $50,8 \mathrm{~cd}$ & $56,3 \mathrm{bcd}$ & $65,8 \mathrm{ab}$ & $76,8 \mathrm{~b}$ & $80,3 \mathrm{~b}$ & $85,0 \mathrm{~b}$ & $89,0 \mathrm{ab}$ & $90,0 \mathrm{ab}$ & $90,8 \mathrm{ab}$ \\
\hline 5. glyphosate + $\mathrm{Sil}^{21}$ & 2.400 & $13,3 \mathrm{~d}$ & $21,8 \mathrm{c}$ & $31,8 \mathrm{~cd}$ & $45,8 \mathrm{~cd}$ & $50,3 \mathrm{~d}$ & $55,5 \mathrm{~cd}$ & $66,5 \mathrm{ab}$ & $74,5 \mathrm{~b}$ & $77,0 \mathrm{~b}$ & $78,8 \mathrm{~b}$ & $85,3 \mathrm{bc}$ & $87,0 \mathrm{~b}$ & $87,8 \mathrm{bc}$ \\
\hline 6. glyphosate + Sil & 3.360 & $16,0 \mathrm{~cd}$ & $22,8 \mathrm{c}$ & $31,8 \mathrm{~cd}$ & $45,0 \mathrm{~cd}$ & $51,3 \mathrm{~cd}$ & $60,0 \mathrm{bc}$ & $67,3 \mathrm{ab}$ & $77,8 \mathrm{ab}$ & $78,8 \mathrm{~b}$ & $83,3 \mathrm{~b}$ & $86,0 \mathrm{bc}$ & $88,3 \mathrm{~b}$ & $88,5 \mathrm{bc}$ \\
\hline 7. glyphosate + Sil & 4.320 & $16,0 \mathrm{~cd}$ & $22,5 \mathrm{c}$ & $34,0 \mathrm{bc}$ & $47,8 \mathrm{c}$ & $59,5 \mathrm{cb}$ & $64,5 \mathrm{ab}$ & $73,0 \mathrm{a}$ & 87,0 a & 90,3 a & 93,5 a & 94,3 a & 94,8 a & 94,8 a \\
\hline 8. imazapyr & 750 & $5,3 \mathrm{e}$ & $9,0 \mathrm{~d}$ & $12,0 \mathrm{e}$ & $18,8 \mathrm{e}$ & $26,3 \mathrm{e}$ & $22,8 \mathrm{e}$ & $32,8 \mathrm{~d}$ & $52,8 \mathrm{c}$ & $57,5 \mathrm{c}$ & $61,3 \mathrm{c}$ & $60,3 \mathrm{~d}$ & $68,3 \mathrm{c}$ & $69,0 \mathrm{~d}$ \\
\hline 9. imazapyr & 1.500 & $6,0 \mathrm{e}$ & $9,0 \mathrm{~d}$ & $9,0 \mathrm{e}$ & $13,23 \mathrm{e}$ & $18,3 \mathrm{e}$ & $19,8 \mathrm{e}$ & $32,3 \mathrm{~d}$ & $53,5 \mathrm{c}$ & $60,0 \mathrm{c}$ & $60,8 \mathrm{c}$ & $58,8 \mathrm{~d}$ & $69,5 \mathrm{c}$ & $69,5 \mathrm{~d}$ \\
\hline 10. diquat & $200+200$ & $45,8 \mathrm{~b}$ & $51,3 \mathrm{~b}$ & $38,5 \mathrm{bc}$ & $67,8 \mathrm{~b}$ & $67,8 \mathrm{~b}$ & $61,0 \mathrm{abc}$ & $53,5 \mathrm{c}$ & $52,5 \mathrm{c}$ & $27,8 \mathrm{~d}$ & $15,5 \mathrm{~d}$ & $11,5 \mathrm{e}$ & $11,0 \mathrm{~d}$ & $6,3 \mathrm{e}$ \\
\hline 11. diquat & $400+400$ & $53,8 \mathrm{a}$ & $59,0 \mathrm{a}$ & $42,0 \mathrm{a}$ & $84,3 \mathrm{a}$ & $80,8 \mathrm{a}$ & $69,0 \mathrm{a}$ & $59,3 \mathrm{bc}$ & $54,0 \mathrm{c}$ & $27,5 \mathrm{~d}$ & $16,0 \mathrm{~d}$ & $12,0 \mathrm{e}$ & $11,0 \mathrm{~d}$ & $6,8 \mathrm{e}$ \\
\hline $\mathrm{F}$ tratamento & & $99,07 * *$ & $110,63 * *$ & $39,86^{* *}$ & $118,54 * *$ & $53,25 * *$ & $60,73 * *$ & $41,08^{* * *}$ & $55,93 * *$ & $105,80 * *$ & $147,29 * *$ & $246,70 * *$ & $387,28 * *$ & $567,63^{* *}$ \\
\hline $\mathrm{CV}(\%)$ & & 17,4 & 13,6 & 16,0 & 10,7 & 13,8 & 12,3 & 13,1 & 10,6 & 9,4 & 9,2 & 7,6 & 6,0 & 5,2 \\
\hline DMS & & 4,85 & 4,84 & 6,18 & 6,40 & 9,18 & 8,31 & 9,92 & 9,37 & 8,19 & 8,02 & 6,65 & 4,47 & 5,13 \\
\hline
\end{tabular}

Médias seguidas de mesma letra, na coluna, não diferem estatisticamente entre si pelo teste $\mathrm{t}(\mathrm{P}>0,05)$; ** valor significativo pelo teste $\mathrm{F}$ a $(\mathrm{P}>0,01)$.

${ }^{1 /} \mathrm{At}=$ Aterbane $0,5 \% \mathrm{v} / \mathrm{v} ;{ }^{2 /}$ Sil $=$ Silwet $0,1 \% \mathrm{v} / \mathrm{v} ; \mathrm{e}^{\mathrm{3l}}$ aplicação seqüencial do herbicida diquat.

A dificuldade no controle de Panicum repens está relacionada com a grande capacidade e rapidez com que as plantas se recuperam, principalmente em função das reservas de carboidratos nos rizomas (Manipura \& Somaratne, 1972). Dessa forma, o fato de o diquat apresentar translocação bastante limitada causa grandes danos principalmente às folhas das plantas, mas permite que as plantas de Panicum repens se recuperem e restabeleçam sua parte aérea.

De maneira geral, os tratamentos com glyphosate proporcionaram o melhor controle, porém este variou em função das doses e dos adjuvantes. As doses de 4.320 g e.a. ha-1 independente dos adjuvantes e de $3.360 \mathrm{~g}$ e.a. hacom Aterbane proporcionaram bom controle: entre 91,3 e $94,8 \%$. Nas outras doses o controle foi menos eficiente (abaixo de 88,5\%). O herbicida imazapyr, independentemente da dose utilizada, apresentou controle abaixo de $69,5 \%$, sendo este considerado insatisfatório na supressão de Panicum repens; o herbicida diquat, apesar das duas aplicações e independentemente da dose utilizada, mostrou-se ineficiente no controle das plantas de Panicum repens.
Na Tabela 3 encontra-se os resultados das avaliações visuais de controle das plantas de Paspalum repens de 2 a 38 DAA. Observa-se aos 2 DAA, para o herbicida glyphosate, uma elevada fitotoxidade - estas plantas apresentavam as folhas bastante danificadas por necroses. Esse fato não era esperado inicialmente, pois trata-se de um produto sistêmico que normalmente apresenta necessidade de um período longo para evidenciar seus sintomas.

Aos 6 DAA, todos os tratamentos com glyphosate atingiram excelente nivel de controle. Aos 12 DAA, as concentrações de 3.360 e 4.320 g e.a. ha-1 com Silwet causaram a morte de todas as plantas. Todos os tratamentos com glyphosate, independentemente da dose e do surfatante utilizado, atingiram $100 \%$ de controle aos 22 DAA, o que demonstrou grande sensibilidade desta espécie a este herbicida.

O herbicida imazapyr aos 2 DAA causou os primeiros sintomas de fitointoxicação, com o início do desenvolvimento de cloroses internervais e arroxeamento das folhas. Aos 38 DAA, quando foi encerrado o estudo, foi observado excelente controle, com a morte de todas as plantas.

Planta Daninha, Viçosa-MG, v. 22, n. 3, p. 453-460, 2004 
Tabela 3 - Avaliações visuais de controle de plantas de Paspalum repens em diferentes épocas após aplicação de herbicidas. Botucatu-SP, 2003

\begin{tabular}{|c|c|c|c|c|c|c|c|c|c|c|c|c|}
\hline \multirow{2}{*}{ Tratamento } & \multirow{2}{*}{\begin{tabular}{|c|} 
Dose \\
$\left(\mathrm{g}\right.$ i/e.a. ha $\left.^{-1}\right)$
\end{tabular}} & \multicolumn{11}{|c|}{ Dias após a aplicação dos herbicidas (DAA) } \\
\hline & & 2 & $6^{-3 /}$ & 9 & 12 & 15 & 18 & 22 & 26 & 30 & 34 & 38 \\
\hline 1. testemunha & - & $0,0 \mathrm{e}$ & $0,0 \mathrm{~d}$ & $0,0 \mathrm{~d}$ & $0,0 \mathrm{~d}$ & $0,0 \mathrm{~d}$ & $0,0 \mathrm{~d}$ & $0,0 \mathrm{~d}$ & $0,0 \mathrm{~d}$ & $0,0 \mathrm{e}$ & $0,0 \mathrm{c}$ & $0,0 \mathrm{c}$ \\
\hline 2. glyphosate $+\mathrm{At}^{1 \mathrm{I}}$ & 2.400 & $69,3 \mathrm{~b}$ & $97,7 \mathrm{a}$ & $99,0 \mathrm{a}$ & 99,7 a & $100,0 \mathrm{a}$ & $100,0 \mathrm{a}$ & $100,0 \mathrm{a}$ & $100,0 \mathrm{a}$ & $100,0 \mathrm{a}$ & $100,0 \mathrm{a}$ & $100,0 \mathrm{a}$ \\
\hline 3. glyphosate + At & 3.360 & $73,0 \mathrm{ab}$ & 98,0 a & $99,5 \mathrm{a}$ & $99,8 \mathrm{a}$ & $100,0 \mathrm{a}$ & $100,0 \mathrm{a}$ & $100,0 \mathrm{a}$ & $100,0 \mathrm{a}$ & $100,0 \mathrm{a}$ & $100,0 \mathrm{a}$ & $100,0 \mathrm{a}$ \\
\hline 4. glyphosate + At & 4.320 & $74,3 \mathrm{ab}$ & $98,3 \mathrm{a}$ & $99,0 \mathrm{a}$ & 99,8 a & $100,0 \mathrm{a}$ & $100,0 \mathrm{a}$ & $100,0 \mathrm{a}$ & $100,0 \mathrm{a}$ & $100,0 \mathrm{a}$ & $100,0 \mathrm{a}$ & $100,0 \mathrm{a}$ \\
\hline 5. glyphosate $+\mathrm{Sil}^{2 /}$ & 2.400 & $70,0 \mathrm{~b}$ & 97,3 a & $98,7 \mathrm{a}$ & $99,0 \mathrm{a}$ & $99,7 \mathrm{a}$ & 99,7 a & $100,0 \mathrm{a}$ & $100,0 \mathrm{a}$ & $100,0 \mathrm{a}$ & $100,0 \mathrm{a}$ & $100,0 \mathrm{a}$ \\
\hline 6. glyphosate + Sil & 3.360 & $70,8 \mathrm{~b}$ & $97,8 \mathrm{a}$ & $99,0 \mathrm{a}$ & $100,0 \mathrm{a}$ & $100,0 \mathrm{a}$ & $100,0 \mathrm{a}$ & $100,0 \mathrm{a}$ & $100,0 \mathrm{a}$ & $100,0 \mathrm{a}$ & $100,0 \mathrm{a}$ & $100,0 \mathrm{a}$ \\
\hline 7. glyphosate + Sil & 4.320 & $78,8 \mathrm{a}$ & $98,0 \mathrm{a}$ & $99,5 \mathrm{a}$ & $100,0 \mathrm{a}$ & $100,0 \mathrm{a}$ & $100,0 \mathrm{a}$ & $100,0 \mathrm{a}$ & $100,0 \mathrm{a}$ & $100,0 \mathrm{a}$ & $100,0 \mathrm{a}$ & $100,0 \mathrm{a}$ \\
\hline 8. imazapyr & 750 & $4,5 \mathrm{de}$ & $16,8 \mathrm{c}$ & $32,5 \mathrm{c}$ & $37,8 \mathrm{c}$ & $66,5 \mathrm{~b}$ & $74,5 \mathrm{~b}$ & $82,8 \mathrm{~b}$ & $90,8 \mathrm{~b}$ & $95,8 \mathrm{~b}$ & 98,5 a & $100,0 \mathrm{a}$ \\
\hline 9. imazapyr & 1.500 & $7,5 \mathrm{~d}$ & $13,8 \mathrm{c}$ & $29,0 \mathrm{c}$ & $40,3 \mathrm{c}$ & $67,8 \mathrm{~b}$ & $73,5 \mathrm{~b}$ & $91,3 \mathrm{~b}$ & $90,0 \mathrm{~b}$ & $94,5 \mathrm{~b}$ & 98,3 a & $100,0 \mathrm{a}$ \\
\hline 10. diquat & $200+200$ & $47,0 \mathrm{c}$ & $35,8 \mathrm{~b}$ & $62,8 \mathrm{~b}$ & $58,0 \mathrm{~b}$ & $56,5 \mathrm{c}$ & $51,5 \mathrm{c}$ & $33,3 \mathrm{c}$ & $17,8 \mathrm{c}$ & $12,8 \mathrm{c}$ & $3,8 \mathrm{~b}$ & $2,0 \mathrm{~b}$ \\
\hline 11. diquat & $400+200$ & $46,3 \mathrm{c}$ & $34,5 \mathrm{~b}$ & $66,25 \mathrm{~b}$ & $55,8 \mathrm{~b}$ & $55,5 \mathrm{c}$ & $48,0 \mathrm{c}$ & $31,3 \mathrm{c}$ & $17,3 \mathrm{c}$ & $5,0 \mathrm{~d}$ & $2,8 \mathrm{~b}$ & $1,8 \mathrm{~b}$ \\
\hline $\mathrm{F}$ tratamento & & $241,01 * *$ & $1.119,61 * *$ & $706,76 * *$ & $266,45^{* *}$ & $488,91 * *$ & $477,61 * *$ & $291,52 * *$ & $4.780,52 * *$ & $3.177,01 * *$ & $3.776,10^{* *}$ & $6.807,90 * *$ \\
\hline $\mathrm{CV}(\%)$ & & 8,2 & 4,0 & 3,8 & 6,0 & 3,8 & 3,9 & 5,7 & 1,6 & 2,1 & 2,0 & 1,54 \\
\hline DMS & & 5,87 & 3,65 & 4,00 & 6,34 & 4,24 & 4,39 & 6,25 & 1,73 & 2,28 & 2,19 & 1,72 \\
\hline
\end{tabular}

Médias seguidas de mesma letra, na coluna, não diferem estatisticamente entre si pelo teste $\mathrm{t}(\mathrm{P}>0,05)$; ** valor significativo pelo teste $\mathrm{F}$ a $(\mathrm{P}>0,01)$.

${ }^{1 /} \mathrm{At}=$ Aterbane $0,5 \% \mathrm{v} / \mathrm{v} ;{ }^{2 /}$ Sil $=$ Silwet $0,1 \% \mathrm{v} / \mathrm{v} ; \mathrm{e}^{\underline{3} /}$ aplicação seqüencial do herbicida diquat.

O herbicida diquat, nas duas doses testadas, apresentou na primeira avaliação uma elevada porcentagem de controle, no qual todas as folhas atingidas pelo herbicida apresentavam necroses, devido provavelmente à baixa translocação e ação de contato desse produto (Oliveira Jr. \& Constantim, 2001; Rodrigues \& Almeida, 1998). Aos 6 DAA, os sintomas começaram a regredir, independentemente da dose testada $(400 \mathrm{e}$ $800 \mathrm{~g}$ i.a. ha ${ }^{-1}$, divididas em aplicações seqüenciais), em função do aparecimento de rebrotas.

Com a aplicação seqüencial (6 DAA) obteve-se novamente um bom nivel de controle, porém novamente as plantas apresentaram rebrotas que inviabilizaram o controle.

De maneira geral, o herbicida glyphosate apresentou resultados excelentes (100\%) para o controle de Paspalum repens, em todas as doses e independentemente do adjuvante testado. Também o herbicida imazapyr mostrou excelente controle desta espécie (100\% para as duas doses), contudo de forma mais lenta que o glyphosate. $O$ herbicida diquat apresentou novamente resultados insatisfatórios.

Planta Daninha, Viçosa-MG, v. 22, n. 3, p. 453-460, 2004
Na Tabela 4 estão apresentados os resultados da avaliação da redução da massa seca das plantas de Panicum repens e Paspalum repens ao final do estudo. Nota-se que todas as doses e herbicidas testados promoveram redução da massa seca das plantas quando comparadas à testemunha; no entanto, essa redução foi dependente dos herbicidas testados.

As plantas de Panicum repens e Paspalum repens submetidas ao herbicida glyphosate, independentemente do tratamento testado, apresentaram as maiores reduções na biomassa das plantas. Observa-se, em termos percentuais, que a dose de $4.320 \mathrm{~g}$ e.a. ha ${ }^{-1}+$ Silwet apresentou a maior redução de biomassa (maior que 50\%).

Para o imazapyr, as duas doses causaram redução inferior à proporcionada pelo glyphosate, caracterizando assim a ineficiência deste produto no controle das plantas de Panicum repens. As parcelas de Paspalum repens tratadas com o herbicida imazapyr apresentaram grande redução de biomassa, com resultados semelhantes aos do glyphosate, porém este apresentou controle mais rápido. Tal fato pode ser explicado pela ação mais 
Tabela 4 - Massa seca das plantas de Panicum repens e Paspalum repens e porcentagem de massa seca ao final do ensaio em relação à testemunha. Botucatu-SP, 2003

\begin{tabular}{|c|c|c|c|c|c|}
\hline \multirow{3}{*}{ Tratamento } & \multirow{3}{*}{$\frac{\text { Dose }}{\left(\mathrm{g} \text { i e.a. } \mathrm{ha}^{-1}\right)}$} & \multicolumn{2}{|c|}{ Panicum repens } & \multicolumn{2}{|c|}{ Paspalum repens } \\
\hline & & \multicolumn{2}{|c|}{ Massa seca } & \multicolumn{2}{|c|}{ Massa seca } \\
\hline & & $(\mathrm{g})$ & $(\%)$ & $(\mathrm{g})$ & $(\%)$ \\
\hline 1. testemunha & - & 832,9 a & 100,0 & $384,2 \mathrm{a}$ & 100,0 \\
\hline 2. glyphosate+Aterbane $0,5 \%$ & 2.400 & $462,7 \mathrm{~cd}$ & 55,6 & $216,0 \mathrm{de}$ & 56,2 \\
\hline 3. glyphosate+Aterbane $0,5 \%$ & 3.360 & $432,1 \mathrm{~d}$ & 51,9 & $196,3 \mathrm{de}$ & 51,1 \\
\hline 4. glyphosate+Aterbane $0,5 \%$ & 4.320 & $425,1 \mathrm{~d}$ & 51,0 & $194,8 \mathrm{de}$ & 50,7 \\
\hline 5. glyphosate+Silwet $0,1 \%$ & 2.400 & $478,8 \mathrm{~cd}$ & 57,5 & $216,0 \mathrm{de}$ & 56,2 \\
\hline 6. glyphosate+Silwet $0,1 \%$ & 3.360 & $433,5 \mathrm{~d}$ & 52,0 & 217,7 cde & 56,7 \\
\hline 7. glyphosate+Silwet $0,1 \%$ & 4.320 & $402,7 \mathrm{~d}$ & 48,3 & $164,3 \mathrm{e}$ & 42,8 \\
\hline 8. imazapyr & 750 & $549,9 \mathrm{bc}$ & 66,0 & $230,1 \mathrm{~cd}$ & 59,9 \\
\hline 9. imazapyr & 1.500 & $565,7 \mathrm{bc}$ & 67,9 & $237,3 \mathrm{bcd}$ & 61,8 \\
\hline 10. diquat & $200+200$ & $624,8 \mathrm{~b}$ & 75,0 & $257,2 \mathrm{bc}$ & 66,9 \\
\hline 11. diquat & $400+400$ & $631,6 \mathrm{~b}$ & 75,8 & $287,4 \mathrm{~b}$ & 74,8 \\
\hline $\mathrm{F}$ tratamento & & $8,09 * *$ & --- & $8,37 * *$ & --- \\
\hline CV $(\%)$ & & 16,4 & --- & 17,4 & -- \\
\hline DMS & & 108,59 & --- & 52,8 & --- \\
\hline
\end{tabular}

Médias seguidas de mesma letra, na coluna, não diferem estatisticamente entre si pelo teste t $(\mathrm{P}>0,1)$.

** valor significativo pelo teste $\mathrm{F}$ a $(\mathrm{P}>0,01)$.

rápida do glyphosate em comparação ao imazapyr, sendo as plantas decompostas rapidamente. O herbicida imazapyr, apesar de interromper o crescimento e causar a morte das regiões meristemáticas logo após a aplicação (Rodrigues \& Almeida, 1998), levou um tempo maior para provocar a morte das plantas.

$\mathrm{O}$ diquat causou pequena redução de massa seca das duas espécies, em razão de o herbicida não atingir provavelmente os rizomas, que possuem reservas de carboidratos, permitindo, assim, a rebrota das plantas (Manipura \& Somaratne, 1974). Não ocorreram diferenças entre as doses testadas, sendo inviável a aplicação deste herbicida no controle de Panicum repens e Paspalum repens.

\section{LITERATURA CITADA}

HOYER, M. V. et al. Florida freshwater plants. Florida: 1996. $256 \mathrm{p}$.

KISSMANN, K. G. Plantas infestantes e nocivas. 2.ed. São Bernardo do Campo: Basf, 1997. t. 1. 824 p.

LORENZI, H. Plantas daninhas do Brasil: terrestres aquáticas, parasitas, tóxicas e medicinais. 3.ed. Nova Odessa: Plantarum, 2000. 440 p.
MANIPURA, W. B.; SOMARATNE, A. Some effects of manual and chemical defoliation on the growth and carboydrate reserves of Panicum repens (L.) Beauv. Weed Res., v. 14, p. 167-172, 1972.

MARTINS, D. Controle de plantas daninhas aquáticas. In: WORKSHOP CONTROLE DE PLANTAS AQUÁTICAS, 1998, Brasília. Resumos... Brasília: IBAMA, 1998. p. $30-31$.

MOREIRA, I. Aspectos taxonômicos e organográficos do Cynodon dactylon (L.) Pers e do Panicum repens L. An. ESALQ., v. 37, p. 103-137, 1977.

NIR, A. Combined control of both weeds and mosquitoes in drainage canals. Phytoparasitica, v. 16, n. 4, p. 355-356, 1988.

OLIVEIRA Jr., R. S.; CONSTANTIN, J. Plantas daninhas e seu manejo. Guaíba: Agropecuária, 2001. 362 p.

PITELLI, R. A. Macrófitas aquáticas no Brasil, na condição de problemáticas. In: WORKSHOP CONTROLE DE PLANTAS AQUÁTICAS, 1998, Brasília. Resumos... Brasília: IBAMA, 1998. p. 12-15.

REDDY, N. K.; SINGH, M. Organosilicone adjuvantes increased the efficacy of glyphosate for control of weeds in citrus (Citrus spp.). Hortiscience, v. 27, n. 9, p. 10031005, 1992.

Planta Daninha, Viçosa-MG, v. 22, n. 3, p. 453-460, 2004 
RODRIGUES, B. N.; ALMEIDA, F. S. Guia de herbicidas. 4.ed. Londrina: 1998. 648 p.

SHILLING, D. G. et al. Influence of surfactants and additives on phytotoxicity of glyphosate to torpedograss. J. Aquatic Plant Manag., v. 28, p. 23-27, 1990.

SOCIEDADE BRASILEIRA DA CIÊNCIA DAS PLANTAS DANINHAS. Procedimentos para instalação, avaliação e análise de experimentos com herbicidas. Londrina: 1995. $42 \mathrm{p}$.
TANAKA, R. H. Prejuízos provocados pelas plantas aquáticas. In: WORKSHOP CONTROLE DE PLANTAS AQUÁTICAS, 1998, Brasília. Resumos... Brasília: IBAMA, 1998. p. 36-38.

VELINI, E. D. Controle de plantas daninhas aquáticas. In: CONGRESSO BRASILEIRO DA CIÊNCIA DAS

PLANTAS DANINHAS, 22., 2000, Foz do Iguaçu.

Palestras... Foz do Iguaçu: Sociedade Brasileira da Ciência das Plantas Daninhas, 2000. p. 137-147. 